DOI: $10.7242 / 2658-705 X / 2020.1 .1$

УДК 539.3

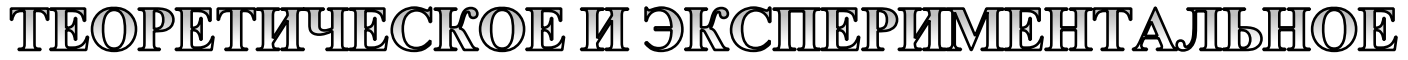

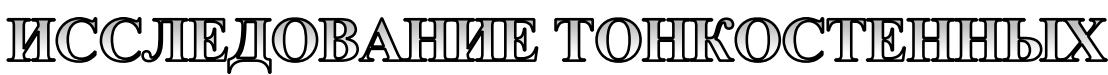

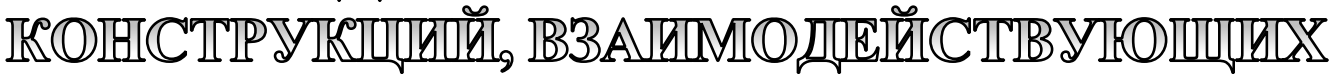

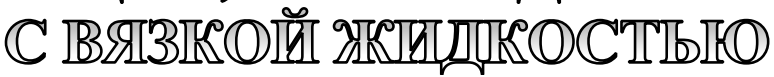

С.А. Бочкарёв, Институт механики сплошных сред УрО РАН

А.О. Каменских, Институт механики сплошных сред УрО РАН

С.В. Лекомцев, Институт механики сплошных сред УрО РАН

А.Н. Сенин, Институт механики сплошных сред УрО РАН

Представлены результаты исследования тонкостенных пластин и цилиндрических оболочек, взаимодействующих с неподвижной или текущей вязкой сжимаемой жидкостью. Численное решение задачи осуществляется с использованием метода конечных элементов. Движение жидкой среды описывается системой линеаризованных уравнений Навье - Стокса, решение которой ищется в виде акустического приближения в терминах потенциала возмущения скорости. Полученные для жидкости соотношения вместе с соответствующими граничными условиями преобразуются с помощью метода Бубнова-Галёркина. Поведение тонкостенной конструкции описывается в рамках классической теории тонких пластин. Математическая постановка задачи динамики упругого тела основана на вариационном принципе возможных перемещений. Оценка устойчивости базируется на вычислении и анализе комплексных собственных значений связанной системы уравнений. Проанализировано влияние вязкости жидкости и других параметров на собственные частоты колебаний и критические скорости потери устойчивости конструкций. C помощью разработанной экспериментальной установки исследованы собственные частоты и соответствующие им декременты гармонических колебаний прямоугольных пластин, расположенных в воздушной среде и на свободной поверхности жидкости. Установлено, что коэффрициент затухания, соответствующий одному типу колебаний (изгибным или крутильным), возрастает с увеличением числа узловых линий. Продемонстрировано, что при взаимодействии пластин с жидкостью данная закономерность может нарушаться.

Ключевые слова: вязкая потенциальная жидкость, тонкостенные конструкиии, устойчивость, собственные колебания, декремент колебаний, МКЭ.

\section{Введение}

При моделировании сложных систем широко распространён подход, согласно которому формализация физических процессов сопровождается принятием ряда гипотез или предположений, упрощаю- щих суть исследуемого явления. Формируемая при этом модель в меньшей степени описывает поведение реального объекта, но в то же время требует более простого математического аппарата для своего анализа и допускает получение явных 
зависимостей, связывающих искомые характеристики с начальными условиями и исследуемыми параметрами системы.

В полной мере вышесказанное относится к задачам исследования деформируемых тонкостенных конструкций, взаимодействующих с неподвижной или текущей жидкой средой. Анализ динамического поведения таких объектов обусловлен их широким применением во многих индустриальных приложениях, в том числе трубопроводном транспорте, ракетно-космическом, авиационном и энергетическом двигателестроении, развиваемых в Пермском крае. $\mathrm{B}$ результате взаимодействия с потоком жидкости могут возникать колебания, приводящие не только к нарушению целостности, но и к полному разрушению конструкции. Очевидно, что для безопасной эксплуатации требуется знание как собственных частот и форм колебаний, так и критических скоростей потока жидкости, вызывающих появление неустойчивости.

Существенный прогресс в решении задач гидроупругости в немалой степени обусловлен моделированием жидкости в виде идеальной потенциальной среды, учётом сжимаемости которой также можно пренебречь. В этом случае её поведение описывается волновым уравнением, допускающим аналитическое решение при определённых условиях, задаваемых на границах контакта жидкости и упругого тела. В рамках этой постановки осуществлено значительное количество исследований, результаты которых изложены в многочисленных публикациях [2, 5, 26, 27].

В том случае, когда свойствами реальных объектов невозможно пренебречь, требуется усложнение как математической модели, так и методов её анализа. В частности, жидкости обладают вязкостью, внутренним трением, вызывающим сопротивление при перемещении их частей относительно друг друга. При построении моделей, исследующих взаимодействие упругих конструкций с потоком жидкости, данным свойством жидких сред, как правило, пренебрегают. В гидродинамике важной величиной, характе- ризующей течение жидкости, является число Рейнольдса, вычисление которого невозможно без учёта вязкости. Поведение вязкой жидкости описывается уравнениями Навье-Стокса, для решения которых в современных коммерческих пакетах используются методы вычислительной гидродинамики (CFD).

Учёт вязкости неподвижной или текущей жидкости в задачах гидроупругости имеет длительную историю и характеризуется использованием различных подходов. Так, в работе [32] решение трёхмерных линеаризованных уравнений Навье Стокса ищется в виде суммы скалярного и векторного потенциалов. Общее решение для системы коаксиальных оболочек, содержащих жидкость, определяется в классе бегущих волн. Аналитическое решение связанных свободных и вынужденных колебаний коаксиальных оболочек, разделённых вязкой жидкостью, описываемой линеаризованными двумерными уравнениями Навье-Стокса, приводится в работе [33]. Продемонстрировано, что размер кольцевого зазора и вязкость жидкости оказывают наиболее значительное влияние на демпфирование колебаний. Аналогичный подход в случае текущей жидкости рассматривается в [13]. Здесь анализируются сложности в реализации условия прилипания, задаваемого на стенке оболочки при моделировании вязкой жидкости. Показано, что для свободно опёртых оболочек влияние нестационарных вязкостных сил возрастает с уменьшением ширины кольцевого канала.

Попытки принять во внимание вязкость жидкости также предпринимались в рамках потенциальной теории. Один из таких подходов [16] основывается на гидравлическом приближении, согласно которому в уравнениях Навье - Стокса вязкостный член заменяется на слагаемое, линейно зависящее от скорости жидкости (модель Дарси). Коэффициентом пропорциональности выступает безразмерный коэффициент трения, который для поверхностей с различной шероховатостью и турбулентного течения может быть вы- 
числен по формуле Никурадзе. Недостатком данного подхода является то, что вязкость жидкости учитывается только через дополнительное слагаемое для составляющей давления, действующей по нормали к смоченной поверхности, оставляя без внимания тангенциальные усилия.

Во втором подходе во внимание принимаются только стационарные силы вязкого сопротивления, полученные из усреднённых по времени уравнений Навье Стокса. В [30] представлена аналитическая модель, в которой движение оболочек описывается с помощью теории тонких оболочек Флюгге. Обобщённые гидродинамические силы определяются в рамках потенциальной теории и вычисляются с помощью преобразования Фурье. Решение задачи осуществляется методом Бубнова - Галёркина. В данной работе для консольных, а в [28] - для жёстко закреплённых оболочек представлено исследование границ устойчивости при различных вариантах течения жидкости и величинах кольцевого зазора между внутренней и внешней оболочкой. Показано, что стационарные силы вязкого сопротивления оказывают существенное влияние на критические скорости течения жидкости.

В рамках аналогичной модели в [29] выполнено исследование влияния на устойчивость ряда системных параметров при кольцевом течении жидкости. В [21] представлена модель, учитывающая стационарные и нестационарные силы вязкого сопротивления, которые определяются из линеаризованных уравнений Навье-Стокса с использованием численной процедуры, основанной на конечноразностном методе. Показано, что эта модель лучше согласуется с экспериментальными данными, представленными в работах $[14,22]$, чем модель, учитывающая только стационарные силы вязкого сопротивления. Исследование колебаний оболочек с неоднородными ограничениями, содержащими внутренний или внутренний кольцевой (внутри области жидкости абсолютно жёсткий стержень) поток как невязкой, так и вязкой жидко- сти, осуществлено в [8, 9]. Для этих целей использован метод Релея - Ритца, в котором в качестве допустимых функций были применены формы колебаний свободно опёртых оболочек в вакууме. Эффективный метод исследования конструкций, содержащих неподвижную вязкую жидкость, предложен в [20].

В работах $[11,12]$ при численном решении задачи, основанном на использовании метода конечных элементов, для случаев невязкой и вязкой жидкости обнаружено существенное расхождение с известными числено-аналитическими решениями для тех случаев, когда потеря устойчивости коаксиальных оболочек осуществляется на высоких модах колебаний. Исследование коаксиальных оболочек, содержащих идеальную или вязкую жидкость, текущую только в кольцевом канале, в том числе с учётом влияния температурных эффектов, представлено в работах [23-25].

Фундаментальное влияние вязкости жидкости в узком канале между двумя цилиндрическими оболочками изучено в [15]. Показано, что с уменьшением числа Рейнольдса значение сил вязкого сопротивления возрастает. Исследование амплитудно-частотных характеристик коаксиальных оболочек с вязким слоем несжимаемой жидкости между ними на резонансных частотах в условиях вибрации осуществлено в [6].

В работе [17] показано, что нелинейные уравнения Навье-Стокса могут быть линеаризованы в акустическом приближении, а соответствующие соотношения записаны в терминах потенциала скорости. В монографии [18] возможности метода были продемонстрированы для различных гидродинамических задач. Несмотря на имеющиеся преимущества, в задачах гидроупругости данный подход не применялся, за исключением тех работ, которые выполнены авторами в рамках проекта РФФИ № 16-41-590646-р_а. Обобщение результатов этих исследований является целью настоящей работы. 


\section{Постановка задачи и основные соотношения}

Объектом исследований является деформируемая тонкостенная конструкция (одиночная пластина или пакет пластин, одиночная цилиндрическая оболочка или две оболочки, вложенные друг в друга), содержащая вязкую сжимаемую жидкость, которая либо неподвижна, либо течёт со скоростью $U$. Необходимо исследовать влияние свойств жидкости на собственные частоты колебаний и границы гидроупругой устойчивости при различных параметрах конструкций.

При моделировании тонкостенного тела предполагается, что его криволинейная поверхность может быть достаточно точно представлена в виде совокупности плоских сегментов [4], в каждом из которых деформации $\bar{\varepsilon}$ определяются в рамках классической теории тонких пластин [31]. Соответствующие соотношения в декартовой системе координат $O \overline{x y z}$, связанной с поверхностью сегмента, имеют вид

$$
\begin{aligned}
& \bar{\varepsilon}=\left\{\varepsilon_{\overline{x x}}, \varepsilon_{\overline{y y}}, \gamma_{\overline{x y}}\right\}^{\mathrm{T}}= \\
& =\left\{\frac{\partial \bar{u}}{\partial \bar{x}}, \frac{\partial \bar{v}}{\partial \bar{y}}, \frac{\partial \bar{u}}{\partial \bar{y}}+\frac{\partial \bar{v}}{\partial \bar{x}}\right\}^{\mathrm{T}}+ \\
& +\bar{z}\left\{-\frac{\partial^{2} \bar{w}}{\partial \bar{x}^{2}},-\frac{\partial^{2} \bar{w}}{\partial \bar{y}^{2}},-2 \frac{\partial^{2} \bar{w}}{\partial \bar{x} \partial \bar{y}}\right\}^{\mathrm{T}} .
\end{aligned}
$$

Здесь и далее прямой чертой сверху обозначены величины, записанные в системе координат $O \overline{x y z}, \bar{u}, \bar{v}$ и $\bar{w}-$ меридиональная, окружная и нормальная компоненты векторов перемещений упругих тел.

Физические соотношения, связывающие вектор обобщённых усилий и моментов $\overline{\mathbf{T}}$ с вектором обобщённых деформаций $\overline{\boldsymbol{\varepsilon}}$, имеют вид

$$
\overline{\mathbf{T}}=\left\{T_{\overline{x x}}, T_{\overline{y y}}, T_{\overline{x y}}, M_{\overline{x x}}, M_{\overline{y y}}, M_{\overline{x y}}\right\}^{\mathrm{T}}=\mathbf{D} \overline{\boldsymbol{\varepsilon}} .
$$

В случае изотропного материала коэффициенты, входящие в матрицу жёсткостей $\mathbf{D}$, определяются известным образом [31] через модель Юнга $E_{s}$ коэффициент Пуассона $v_{s}$.
Для описания движения вязкой сжимаемой жидкости рассматривается система линеаризованных уравнений Навье - Стокса

$$
\begin{aligned}
& \rho_{f} \frac{\partial \mathbf{v}}{\partial t}=-\nabla p+\frac{v_{f} \rho_{f}}{3} \nabla \nabla \mathbf{v}+v_{f} \rho_{f} \Delta \mathbf{v}, \\
& \frac{\partial \rho}{\partial t}+\rho_{f} \nabla \cdot \mathbf{v}=0, \quad \frac{\partial p}{\partial \rho}=c_{f}^{2},
\end{aligned}
$$

где $\mathbf{v}$ - вектор скорости; $p, \rho$ и $v_{f}$ - давление, плотность и кинематическая вязкость жидкости; $\rho_{f}$ - плотность жидкости в состоянии покоя; $c_{f}-$ скорость звука в жидкости; $t$ - время; $\nabla$ и $\Delta$ - дифференциальные операторы Набла и Лапласа.

Компоненты тензора напряжений $F_{i j}$ в вязкой жидкости определяются соотношением

$$
\begin{aligned}
& F_{i j}=\left(-p-\frac{2}{3} v_{f} \rho_{f} \nabla \cdot \mathbf{v}\right) g_{i j}+ \\
& +v_{f} \rho_{f}\left(\nabla_{i} \mathbf{v}^{j}+\nabla_{j} \mathbf{v}^{i}\right),
\end{aligned}
$$

где $g_{i j}-$ метрический тензор.

Осуществляя решение системы (3) в виде $\mathbf{v}=\nabla \phi$, где $\phi-$ потенциал скорости, получим систему уравнений теории вязкой потенциальной жидкости [17-18]

$$
\begin{aligned}
& {\left[\left(1+\frac{4}{3} \frac{v_{f}}{c_{f}^{2}} \frac{\partial}{\partial t}\right) \Delta-\frac{1}{c_{f}^{2}} \frac{\partial^{2}}{\partial t^{2}}\right] \phi=0,} \\
& p=\rho_{f}\left(\frac{4}{3} v_{f} \Delta-\frac{\partial}{\partial t}\right) \phi .
\end{aligned}
$$

На входе в конструкцию и выходе из неё потенциал скорости подчиняется следующим граничным условиям:

$$
x=0: \quad \phi=0 ; \quad x=L: \quad \partial \phi / \partial x=0,
$$

где $L$ - длина конструкции. На смоченной поверхности $S_{\sigma}=S_{s} \cap S_{f}$ задаётся условие прилипания [3]

$$
\overline{\mathbf{P}}=\overline{\mathbf{F}}, \quad \frac{\partial \mathbf{u}}{\partial t}=\nabla \phi,
$$

где $S_{s}$ и $S_{f}$ - поверхности, ограничивающие объёмы упругого тела $V_{s}$ и жидкости $V_{f}$ соответственно; $\overline{\mathbf{P}}$ и $\overline{\mathbf{F}}-$ векторы напряжений в упругом теле и жидкости на границе контакта; 
$\overline{\mathbf{u}}=\left\{\bar{u}, \bar{v}, \bar{w}, \theta_{\bar{x}}, \theta_{\bar{y}}, \theta_{\bar{z}}\right\}^{\mathrm{T}}-$ вектор перемещений и углов поворота вокруг соответствующих осей.

В случае вязкой жидкости, текущей со скоростью $U$ в направлении $O x$, уравнения (5) и (7) приводятся к единой с упругим телом системе координат

$$
\begin{aligned}
& \Delta \phi+\Theta\left(\frac{\partial}{\partial t}+U \frac{\partial}{\partial x}\right) \Delta \phi- \\
& -\frac{1}{c_{f}^{2}}\left(\frac{\partial^{2}}{\partial t^{2}}+2 U \frac{\partial^{2}}{\partial t \partial x}+U^{2} \frac{\partial^{2}}{\partial x^{2}}\right) \phi=0,(8) \\
& \Theta=\frac{4}{3} \frac{v_{f}}{c_{f}^{2}} . \\
& \quad p=\rho_{f}\left(\frac{4}{3} v_{f} \Delta-\frac{\partial}{\partial t}-U \frac{\partial}{\partial x}\right) \phi \\
& \left\{P_{\bar{x}}, P_{\bar{y}}, P_{\bar{z}}\right\}^{\mathrm{T}}=\left\{F_{\overline{z x}}, F_{\overline{z y}},-F_{\overline{z z}}\right\}^{\mathrm{T}} \\
& \frac{\partial \mathbf{u}}{\partial t}+U \frac{\partial \mathbf{u}}{\partial x}=\nabla \phi, \\
& F_{\overline{z x}}=\Xi \frac{\partial^{2} \phi}{\partial \bar{z} \partial \bar{x}}, \quad F_{\overline{z x}}=\Xi \frac{\partial^{2} \phi}{\partial \bar{z} \partial \bar{y}} \\
& F_{\overline{z x}}=-\left(p+\frac{\Xi}{3} \Delta \phi\right)+\Xi \frac{\partial^{2} \phi}{\partial \bar{z}^{2}} \\
& \Xi=2 v_{f} \rho_{f} .
\end{aligned}
$$

Уравнение потенциала возмущения скорости (8) вместе с граничными условиями (6) и (10) преобразуются к слабой форме с помощью метода БубноваГалёркина. Математическая формулировка задачи динамики упругого тонкостенного тела основана на вариационном принципе возможных перемещений, который учитывает работу сил инерции и нормальные и тангенциальные компоненты сил, действующих со стороны жидкости (11).

При численном решении задачи используется метод конечных элементов (МКЭ), стандартные операции которого позволяют получить связанную систему уравнений, описывающую взаимодействие упругого тела и жидкости, которая в матричной форме имеет следующий вид:

$$
\begin{aligned}
& {\left[\begin{array}{cc}
\mathbf{M}_{s} & 0 \\
\mathbf{M}_{f s}^{v} & \mathbf{M}_{f}
\end{array}\right]\left\{\begin{array}{l}
\ddot{\mathbf{u}} \\
\ddot{\boldsymbol{\phi}}
\end{array}\right\}+} \\
& +\left[\begin{array}{cc}
\mathbf{C}_{s}^{v} & \mathbf{C}_{s f} \\
\mathbf{C}_{f s}+\mathbf{C}_{f s}^{v} & \mathbf{C}_{f}+\mathbf{C}_{f}^{v}
\end{array}\right]\left\{\begin{array}{l}
\dot{\mathbf{u}} \\
\dot{\boldsymbol{\phi}}
\end{array}\right\}+ \\
& +\left[\begin{array}{cc}
\mathbf{K}_{s} & \mathbf{K}_{s f}^{v} \\
0 & \mathbf{K}_{f}
\end{array}\right]+ \\
& \left.+\left[\begin{array}{cc}
\mathbf{A}_{s}^{v} & \mathbf{A}_{s f} \\
\mathbf{A}_{f s}+\mathbf{A}_{f s}^{v} & \mathbf{A}_{f}+\mathbf{A}_{f}^{v}
\end{array}\right]\right)\left\{\begin{array}{l}
\mathbf{u} \\
\phi
\end{array}\right\}=0,
\end{aligned}
$$

где и и $\phi-$ обобщённые векторы перемещений и углов поворота тонкостенной конструкции и потенциала возмущения скорости, точка сверху обозначает дифференцирование по времени. Матрицы масс $\mathbf{M}$, демпфирования $\mathbf{C}$, жёсткости $\mathbf{K}$ и гидродинамической жёсткости $\mathbf{A}$ для отдельных конечных элементов формируются следующим образом:

$$
\begin{aligned}
& \overline{\mathbf{K}}_{s}=\int_{V_{s}} \mathbf{B}^{\mathrm{T}} \mathbf{D} \mathbf{B} \mathrm{d} V, \\
& \overline{\mathbf{M}}_{s}=\int_{V_{s}} \rho_{s} \mathbf{N}^{\mathrm{T}} \mathbf{N} \mathrm{d} V, \\
& \overline{\mathbf{C}}_{s f}=\int_{S_{\sigma}} \rho_{f} \mathbf{N}_{\overline{\mathrm{w}}}^{\mathrm{T}} \mathbf{F} \mathrm{d} S, \\
& \overline{\mathbf{A}}_{s f}=\int_{S_{\sigma}} \rho_{f} U \mathbf{N}_{\bar{w}}^{\mathrm{T}} \frac{\partial \mathbf{F}}{\partial x} \mathrm{~d} S, \\
& \overline{\mathbf{C}}_{s}^{v}=\Xi \int_{S_{\sigma}}\left\{\frac{\partial \mathbf{N}_{\bar{u}}}{\partial \bar{x}}, \frac{\partial \mathbf{N}_{\bar{v}}}{\partial \bar{y}}\right\}^{\mathrm{T}} \mathbf{N}_{\bar{w}} \mathrm{~d} S, \\
& \overline{\mathbf{A}}_{s}^{v}=\Xi \int_{S_{\sigma}} U\left\{\frac{\partial \mathbf{N}_{\bar{u}}}{\partial \bar{x}}, \frac{\partial \mathbf{N}_{\bar{v}}}{\partial \bar{y}}\right\}^{\mathrm{T}} \frac{\partial \mathbf{N}_{\bar{w}}}{\partial \bar{x}} \mathrm{~d} S, \\
& \overline{\mathbf{K}}_{s f}^{v}=\int_{S_{\sigma}}\left(\frac{\partial \mathbf{N}_{\bar{w}}^{\mathrm{T}}}{\partial \bar{x}} \frac{\partial \mathbf{F}}{\partial \bar{x}}+\frac{\partial \mathbf{N}_{\bar{w}}^{\mathrm{T}}}{\partial \bar{y}} \frac{\partial \mathbf{F}}{\partial \bar{y}}\right) \mathrm{d} S, \\
& \mathbf{K}_{f}=\int_{V_{f}}\left(\frac{\partial \mathbf{F}^{\mathrm{T}}}{\partial x} \frac{\partial \mathbf{F}}{\partial x}+\frac{\partial \mathbf{F}^{\mathrm{T}}}{\partial y} \frac{\partial \mathbf{F}}{\partial y}+\frac{\partial \mathbf{F}^{\mathrm{T}}}{\partial \mathbf{z}} \frac{\partial \mathbf{F}}{\partial \mathbf{z}}\right) \mathrm{d} V, \\
& \mathbf{M}_{f}=\int_{V_{f}} \frac{1}{\mathbf{C}_{f}^{2}} \mathbf{F}_{f}^{\mathrm{T}} \mathbf{F} \mathrm{d} V, \\
& \int_{f} \frac{2 U}{c_{f}^{2}} \mathbf{F}^{\mathrm{T}} \frac{\partial \mathbf{F}}{\partial x} \mathrm{~d} V,
\end{aligned}
$$




$$
\begin{aligned}
& \mathbf{A}_{f}=\int_{V_{f}} \frac{U^{2}}{c_{f}^{2}} \frac{\partial \mathbf{F}^{\mathrm{T}}}{\partial x} \frac{\partial \mathbf{F}}{\partial x} \mathrm{~d} V, \\
& \overline{\mathbf{C}}_{f s}=-\int_{S_{\sigma}} \mathbf{F}^{\mathrm{T}} \mathbf{N}_{\bar{w}} \mathrm{~d} S, \\
& \overline{\mathbf{A}}_{f s}=-\int_{S_{\sigma}} U \frac{\partial \mathbf{F}^{\mathrm{T}}}{\partial x} \mathbf{N}_{\bar{w}} \mathrm{~d} S, \\
& \overline{\mathbf{C}}_{f s}^{v}=\Theta \int_{S_{\sigma}} U\left(\frac{\partial \mathbf{F}^{\mathrm{T}}}{\partial x} \mathbf{N}_{\bar{w}}-\mathbf{F}^{\mathrm{T}} \frac{\partial \mathbf{N}_{\bar{w}}}{\partial x}\right) \mathrm{d} S, \\
& \mathbf{C}_{f}^{v}=\Theta \int_{V_{f}}\left(\frac{\partial \mathbf{F}^{\mathrm{T}}}{\partial x} \frac{\partial \mathbf{F}}{\partial x}+\frac{\partial \mathbf{F}^{\mathrm{T}}}{\partial y} \frac{\partial \mathbf{F}}{\partial y}+\frac{\partial \mathbf{F}^{\mathrm{T}}}{\partial z} \frac{\partial \mathbf{F}}{\partial z}\right) \mathrm{d} V, \\
& \overline{\mathbf{A}}_{f s}^{v}=\Theta \int_{S_{\sigma}} U^{2} \frac{\partial \mathbf{F}^{\mathrm{T}}}{\partial x} \frac{\partial \mathbf{N}_{\bar{w}}}{\partial x} \mathrm{~d} S, \\
& \mathbf{A}_{f}^{v}=-\Theta \int_{V_{f}} U\left(\frac{\partial^{2} \mathbf{F}^{\mathrm{T}}}{\partial x^{2}} \frac{\partial \mathbf{F}}{\partial x}+\frac{\partial^{2} \mathbf{F}^{\mathrm{T}}}{\partial x \partial y} \frac{\partial \mathbf{F}}{\partial y}+\frac{\partial^{2} \mathbf{F}^{\mathrm{T}}}{\partial x \partial z} \frac{\partial \mathbf{F}}{\partial z}\right) \mathrm{d} V, \\
& \overline{\mathbf{M}}_{f s}^{v}=-\Theta \int_{S_{\sigma}} \mathbf{F}^{\mathrm{T}} \mathbf{N}_{\bar{w}} \mathrm{~d} S .
\end{aligned}
$$

Здесь В - матрица градиентов, устанавливающая связь между вектором деформаций и вектором узловых перемещений конечного элемента тонкостенного тела; $\mathbf{F}, \mathbf{N}, \mathbf{N}_{\bar{u}}, \mathbf{N}_{\bar{v}}$ и $\mathbf{N}_{\bar{w}}-$ функции формы для потенциала возмущения скорости, обобщённого вектора узловых перемещений упругой конструкции и его составляющих; $\rho_{s}-$ плотность материала упругого тела.

Часть матриц в соотношении (12) формируется в координатах $(\bar{x}, \bar{y}, \bar{z})$. Для получения решения в глобальной системе координат Oxyz, в которой заданы геометрия и сформулированы определяющие соотношения, выполняется преобразование с помощью матрицы направляющих косинусов [1].

С учётом представления возмущённого движения упругого тела и жидкой среды в виде $\{\mathbf{u}, \phi\}=\{\tilde{\mathbf{u}}, \tilde{\phi}\} \exp (\lambda t)$, где $\tilde{\mathbf{u}}$ и $\tilde{\phi}$ - некоторые функции координат, исходная система уравнений (12) сводится к обобщённой задаче на собственные значения следующего вида:

$$
\begin{aligned}
& \lambda\left[\begin{array}{cc}
\mathbf{M} & 0 \\
0 & \mathbf{I}
\end{array}\right]\left\{\begin{array}{c}
\lambda \mathbf{x} \\
\mathbf{x}
\end{array}\right\}+\left[\begin{array}{cc}
\mathbf{C} & \mathbf{K}+\mathbf{A} \\
-\mathbf{I} & 0
\end{array}\right]\left\{\begin{array}{c}
\lambda \mathbf{x} \\
\mathbf{x}
\end{array}\right\}=0, \\
& \mathbf{x}=\left\{\begin{array}{l}
\mathbf{u} \\
\phi
\end{array}\right\},
\end{aligned}
$$

здесь $\lambda=\delta+\mathrm{i} \omega-$ характеристический показатель $(\mathrm{i}=\sqrt{-1})$, в котором $\omega-$ собственная частота колебаний, а $\delta$ - величина, отвечающая за демпфирование системы; I - единичная матрица.

Задача оценки устойчивости системы, описывающей взаимодействие упругого тела и потока жидкости, сводится к нахождению и анализу характеристического показателя $\lambda$ при последовательном увеличении скорости потока жидкости. Для вычисления комплексных собственных значений $\lambda$ системы уравнений (13) применяется алгоритм, в основе которого лежит неявно перезапускаемый метод Арнольди [19].

В случае если конструкция состоит из нескольких компонент (две пластины или оболочки, два объёма жидкости), система (12) дополняется соответствующими уравнениями, а отдельные подматрицы формируются аналогичным образом.

На краях упругих тел могут быть заданы граничные условия жёсткой заделки (C, $u=v=w=\theta_{x}=\theta_{y}=\theta_{z}=0$ ), шарнирного опирания (S, $v=w=0)$ или свободного края (F).

\section{Пластины, контактирующие с жидкостью}

В некоторых отраслях промышленности определённый практический интерес представляют конструкции, расположенные на поверхности жидкости и контактирующие с ней только с одной стороны (плавающие льдины, плавучие аэродромы и платформы). Их упрощённый аналог, представляющий собой тонкую пластину, исследован с помощью разработанной экспериментальной установки; определены собственные частоты и декременты гармонических колебаний в условиях резонанса, выявлены свойственные им качественные и количественные закономерности. Необ- 
ходимые для проведения эксперимента некоторые предварительные вычисления были сделаны с помощью изложенных в предыдущем разделе математического аппарата и конечно-элементного алгоритма.

Экспериментальная установка (рис. 1) представляет собой толстостенный призматический бак, вместо верхней крышки которого установлена тонкая пластина длиной $a$, шириной $b$ и толщиной $h$. В конструкции предусмотрена возможность зажимать или оставлять свободным любой из четырёх краёв образца, что позволяет анализировать различные варианты закрепления. Возбуждение колебаний осуществляется электромагнитным полем. С этой целью на пластину приклеивается лёгкий неодимовый магнит, над которым на П-образной раме позиционируется катушка. На неё с генератора сигналов через усилитель подаётся переменной ток заданной частоты или прямоугольный импульс. Возникающая электромагнитная сила воздействует на магнит и приводит пластину в движение.

Такой способ возбуждения вносит незначительную дополнительную массу в систему, однако сосредотачивает силовое воздействие в заранее известной области. Его достоинством также является возможность исследования образцов из материалов, не обладающих магнитными свойствами. Бак устанавливается на металлический постамент, горизонтальное положение которого регулируется с помощью подъёмных ножек и лазерного угломера, и заполняется жидкостью вплоть

до контакта с нижней поверхностью пластины. Специально предусмотренные в стенках бака технологические отверстия позволяют откачивать излишки жидкости в случае необходимости.

Исследование собственных частот и декрементов гармонических колебаний пластины осуществляется с использованием метода лазерной виброметрии. Снятие показаний проводится цифровым виброметром Polytec PDV-100. Положение точек на поверхности образца, в которых осуществляется возбуждение и проводятся измерения, находится на основе результатов численного решения соответствующей модальной задачи с помощью метода конечных элементов [10] и задачи оптимизации. Собственные частоты колебаний пластины определяются из Фурье-образа сигнала, полученного после возмущения образца кратковременным прямоугольным импульсом длительностью 0,5 мс. Для возбуждения гармонических колебаний на катушку с генератора сигналов через усилитель мощности подаётся переменный ток с частотой, равной собственной частоте колебаний пластины. После выхода на установившийся режим электрическая цепь размыкается и наблюдаются затухающие гармонические колебания. Их декремент $\delta$ находится по полученной в результате измерений виброграмме после аппроксимации её огибающей c помощью экспоненциальной функции.

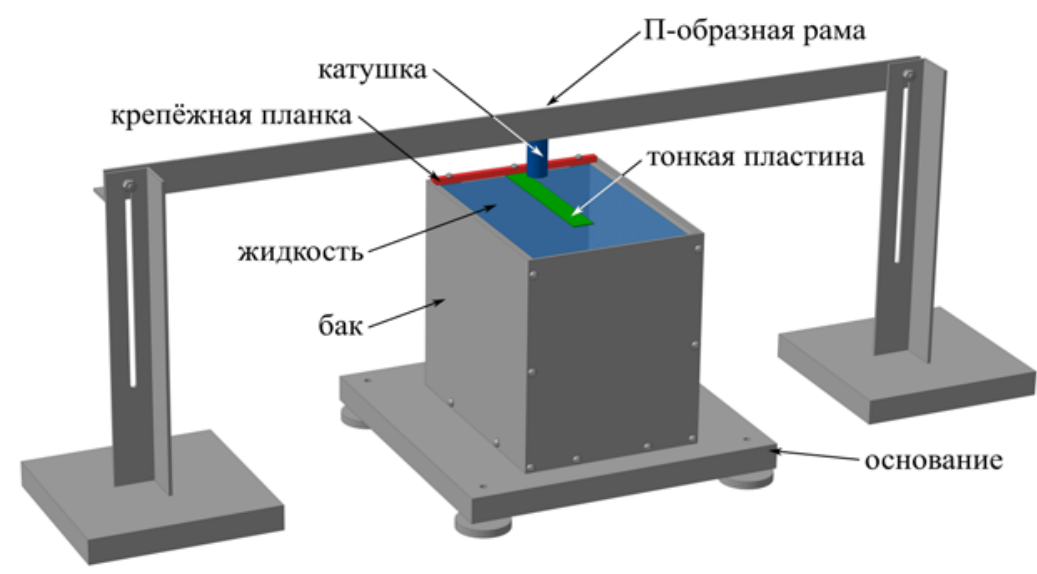

Рис. 1. Трёхмерная модель экспериментальной установки 
Рассмотренные варианты закрепления пластин и их расположение схематично изображены на рис. 2. В ходе численных экспериментов установлено, что их собственные формы колебаний качественно не изменяются при взаимодействии с жидкостью. Они остаются такими же, с точностью до знака, но иногда возможно незначительное смещение положения узловых линий. Влияние дополнительной массы (присоединённого магнита) на первые восемь собственных частот колебаний исследуемых образцов в воздушной среде не превышает относительной разницы, равной $3 \%$. При взаимодействии с жидкостью эта величина снижается до $1 \%$.

Экспериментальные исследования проводились в два этапа. Сначала определялись собственные частоты колебаний пластин. Затем полученная информация использовалась для их возбуждения гармонической электромагнитной силой в условиях резонанса. Аппроксимация огибающих с помощью экспоненциальной функции вида $y(t)=A e^{-\delta t}$ позволяет количественно оценить скорость затухания колебаний.

Анализ результатов позволил сделать следующие выводы:

- собственные частоты колебаний рассмотренных пластин снижаются на 30$55 \%$ при их взаимодействии с жидкостью;

- декремент $\delta$, соответствующий одному типу колебаний (изгибные или крутильные), возрастает с увеличением числа узловых линий. Однако данная особенность может нарушаться, если пластина контактирует с жидкостью;

- при одинаковой возбуждающей силе амплитуда колебаний снижается бо- лее чем в десять раз при взаимодействии пластины с жидкостью;

- численно определённые собственные частоты колебаний согласуются с экспериментальными данными в пределах относительной погрешности, равной $5 \%$.

В ходе исследований установлено, что при колебаниях консольной пластины (рис. 2, вариант 1) на низшей частоте $\omega_{1}$ на свободной поверхности жидкости возникают волны, которые отражаются от стенок бака и движутся в обратном направлении. Это приводит к образованию стоящих волн большой амплитуды, которые искажают огибающую затухающих колебаний вплоть до того, что её становится невозможно аппроксимировать экспонентой. С увеличением частоты возбуждения амплитуда волн снижается. На частоте 50 Гц они становятся мало заметными, а свыше 100 Гц их влияние уже совсем несущественно. Известно, что с увеличением вязкости жидкости скорость затухания гидроупругих колебаний возрастает. Для демонстрации этого эффекта была проведена серия экспериментов со смесью глицерина и воды разной концентрации. На рис. 3 изображены огибающие гармонических колебаний консольной пластины (рис. 2, вариант 1), полученные для разных растворов. Объёмная доля глицерина указана в процентах $(0 \%$ соответствует чистой воде). Во время проведения исследований было замечено, что с увеличением вязкости жидкости снижается амплитуда поверхностных волн, возникающих на низшей частоте. Ступенчатый вид огибающей постепенно сглаживается, и при концентрации глицерина 50\% кривая может быть достоверно описана экспоненциальной зависимостью (рис. 3, $a$ ).

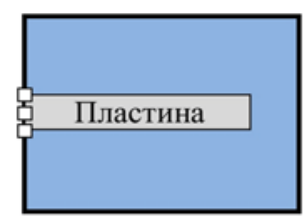

Вариант 1, CFFF

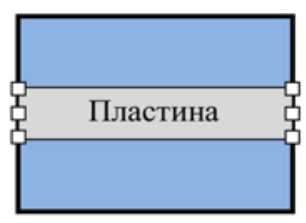

Вариант 2, CFCF

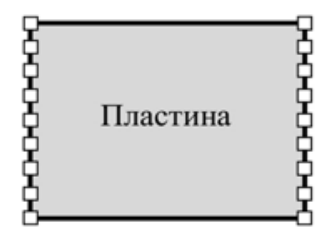

Вариант 3, CFCF

Рис. 2. Расположение экспериментальных образцов и схемы их закрепления 

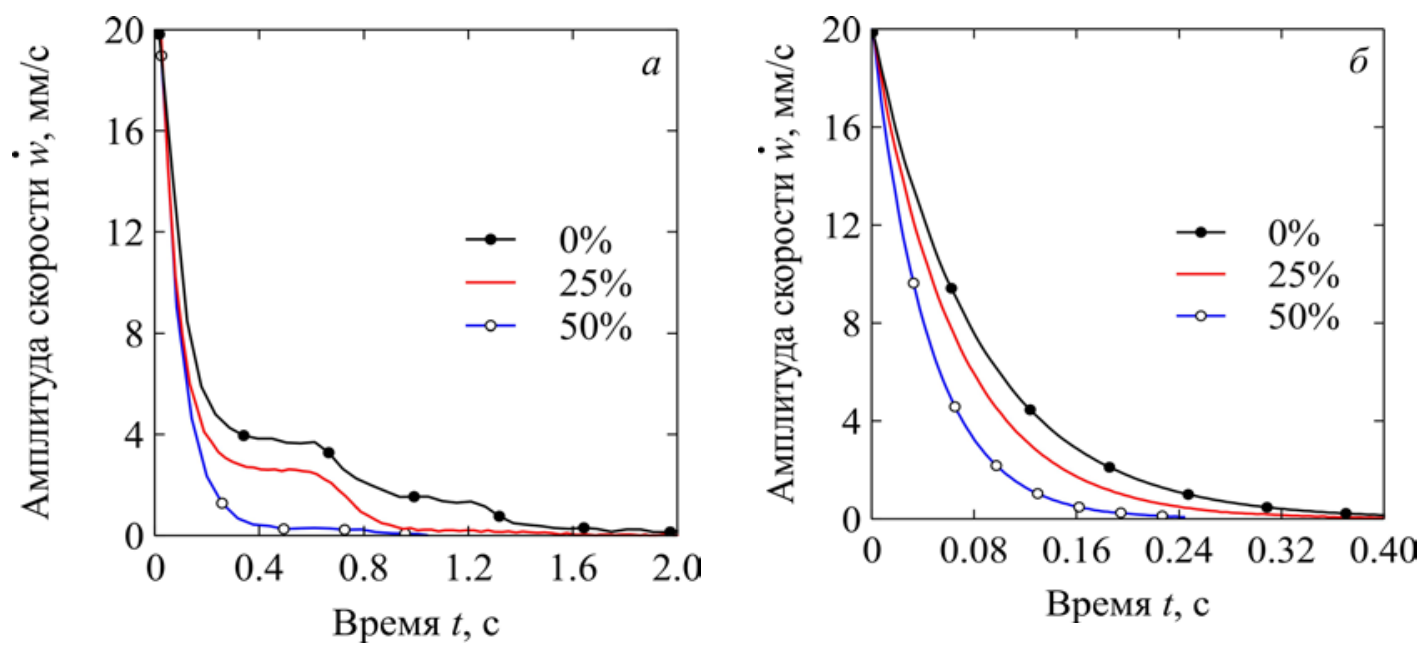

Рис. 3. Огибающие затухающих гармонических колебаний пластины, расположенной на свободной поверхности смеси глищерина и воды разной конщентращии: $a-\omega_{1}, \sigma-\omega_{5}$

\section{Оболочки, взаимодействующие с жидкостью}

Рассматривается цилиндрическая оболочка со средним радиусом $R$, длиной $L$ и толщиной $h$, содержащая внутренний поток жидкости.

Для представления результатов расчётов, полученных для труб с жидкостью, вводятся коэффициенты демпфирования $\zeta$ и $\xi$, используемые в системах с наличием и отсутствием гидродинамического демпфирования или в случае учёта сжимаемости жидкости

$$
\xi=\left[\frac{\delta}{\omega}\right] \times 10^{7}, \quad \zeta=\left[\frac{\left|\lambda_{1}\right|}{\left|\lambda_{2}\right|}\right] \times 10^{8} .
$$

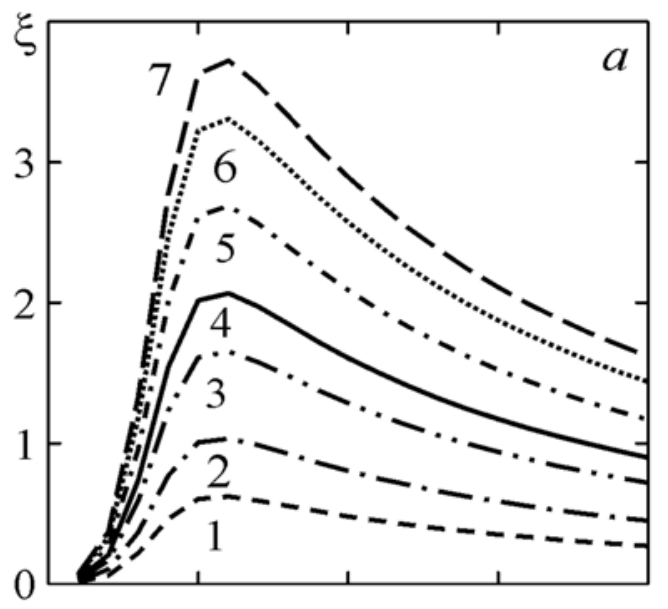

На рис. 4, $a$ представлено изменение коэффициентов демпфирования $\xi$ от номера гармоники в окружном направлении j при жёстком закреплении трубы на обоих краях (СС) и различных значениях кинематической вязкости $v_{f}$ (число волн в меридиональном направлении $m=1$ ) несжимаемой жидкости. Из представленных данных следует, что коэффициент демпфирования возрастает с увеличением вязкости и имеет ярко выраженный локальный максимум, не зависящий от значения вязкости. На рис. 4, б приведены коэффициенты демпфирования, полученные для трубы с различными граничными условия-

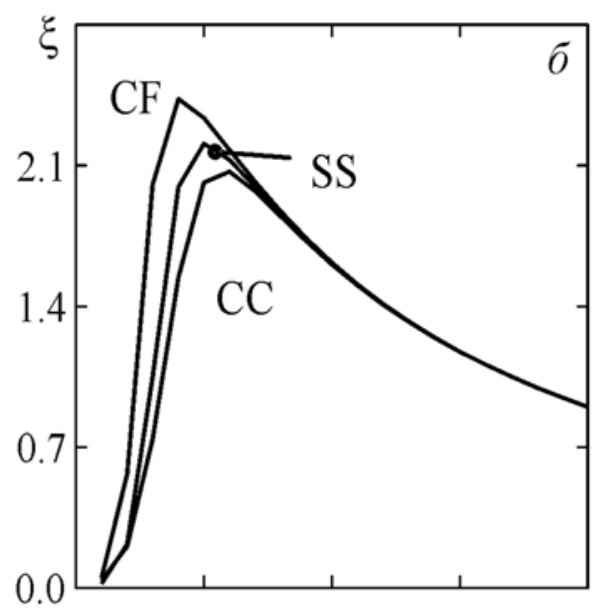

Рис. 4. Изменение коэффициента демпфирования $\xi$ от номера гармоники для ичилиндрической оболочки с неподвижной вязкой жидкостью при различных значениях кинематической вязкости $v_{f}($ () и и граничных условиях (б):

$a-C C, 1-v_{f}=3 \times 10^{-7}, 2-v_{f}=5 \times 10^{-7}, 3-v_{f}=8 \times 10^{-7}, 4-v_{f}=1 \times 10^{-6}, 5-v_{f}=1,3 \times 10^{-6}$, $6-v_{f}=1,6 \times 10^{-6}, 7-v_{f}=1,8 \times 10^{-6} ; \sigma-v_{f}=1 \times 10^{-6}$ 
ми. Эти результаты демонстрируют, что вязкость жидкости оказывает наиболее сильное влияние на менее жёсткие системы, а различие в граничных условиях проявляется только на низших гармониках.

На рис. 5, $a$ показаны зависимости коэффициентов демпфирования $\xi$ от номера гармоники $j$, полученные для трубы, жёстко закреплённой на обоих краях при различных значениях скорости течения жидкости $U$ и фиксированном значении кинематической вязкости $v_{f}$. Из этих данных видно, что существенное изменение коэффициента демпфирования имеет место только в том случае, когда скорость течения жидкости достигает значительной величины. Влияние вязкости в случае консольно закреплённой трубы (CF), содержащей текущую жидкость, показано на рис. 5, б. Здесь также имеет место рост коэффициента демпфирования $\zeta$ при увеличении вязкости жидкости, но влияние вязкости носит локализованный характер и ограничено окрестностями гармоники, на которой осуществляется потеря устойчивости.

Выше было отмечено, что в работе [13] обсуждаются сложности в реализации условия прилипания на границе упругого тела и текущей вязкой жидкости. В этом случае скорость жидко- сти на смоченной поверхности должна быть равна нулю. При этом центробежная компонента гидродинамического давления, влияющая на возникновение неустойчивости, также становится равной нулю, что противоречит физической природе явления. В качестве одного из возможных решений в [13] предлагается на границе контакта задавать осреднённое значение скорости $U_{a v}$, которое для случая цилиндрической оболочки имеет вид [7]

$$
U_{a v}=U \frac{2 s^{2}}{(s+1)(2 s+1)},
$$

где параметр $s$ зависит от числа Рейнольдса.

На рис. 6 представлены зависимости безразмерной критической скорости $\Lambda=U\left[\rho_{s}\left(1-v_{s}^{2}\right) / E_{s}\right]^{0,5}$ от отношений длины и толщины оболочки к её радиусу $L / R$ (рис. $6, a)$ и $h / R$ (рис. $6, \sigma$ ). В обоих случаях радиус оболочки фиксируется, а длина или толщина варьируются в некотором диапазоне. Представленные данные демонстрируют, что учёт вязкости жидкой среды приводит к росту границ гидроупругой устойчивости. Это качественно согласуется с результатами работы [13], а количественные расхождения могут быть объяснены как неопределён-

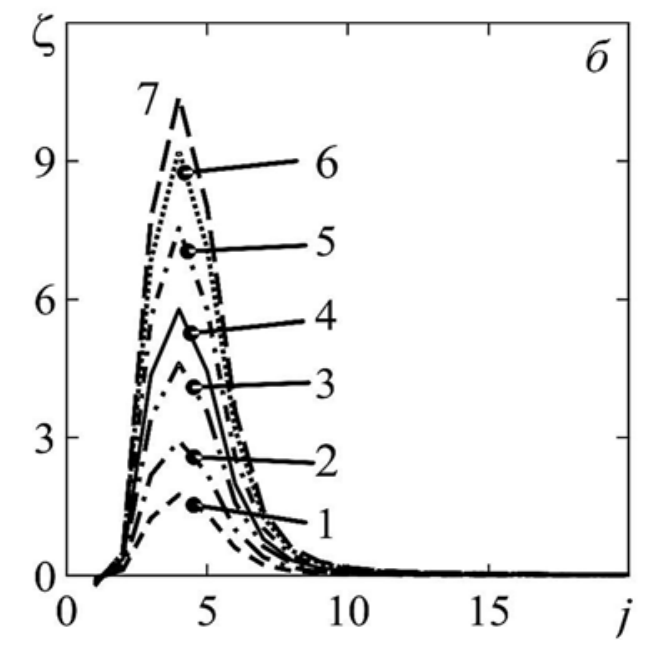

Рис. 5. Изменение коэффициентов демпфирования и от номера гармоники для ичлиндрической оболочки с текущей вязкой жидкостью при различных значениях (a) скорости течения жидкости $U(\mathrm{~s} / \mathrm{c})$ и (б) кинематической вязкости $v_{f}$ : $a-C C, 1-v_{f}=1 \times 10^{-6} ; \sigma-C F, U=300 \mathrm{M} / \mathrm{c}, 1-v_{f}=3 \times 10^{-7}, 2-v_{f}=5 \times 10^{-7}$, $3-v_{f}=8 \times 10^{-7}, 4-v_{f}=1 \times 10^{-6}, 5-v_{f}=1,3 \times 10^{-6}, 6-v_{f}=1,6 \times 10^{-6}, 7-v_{f}=1,8 \times 10^{-6}$ 

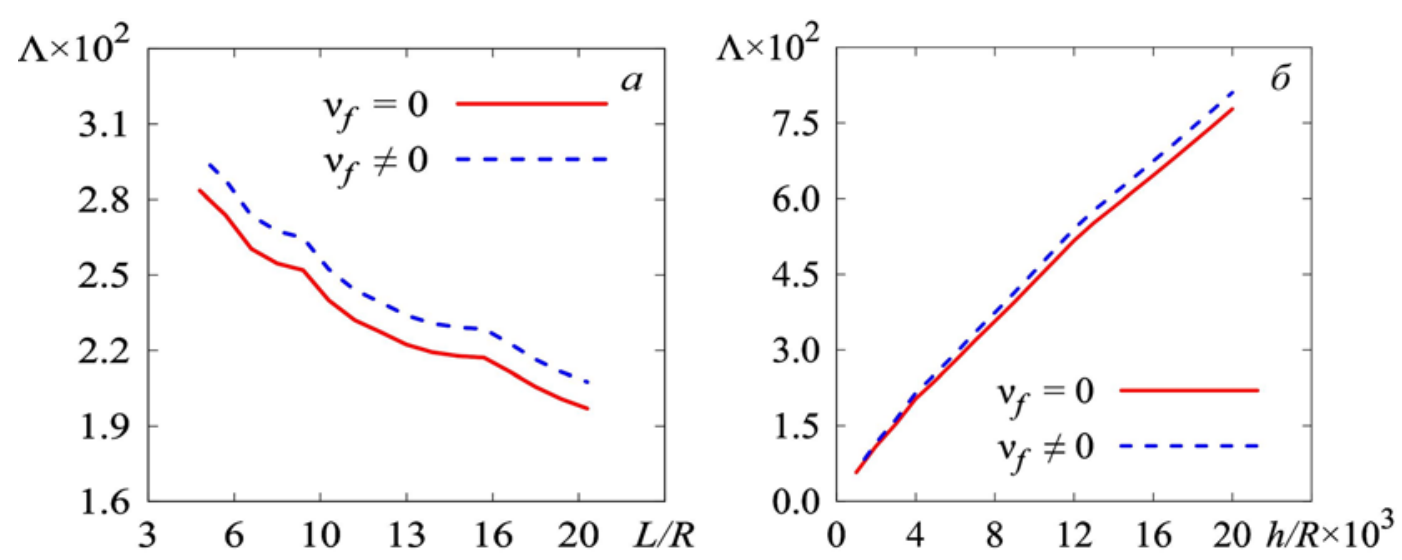

Рис. 6. Зависимости безразмерной критической скорости $\Lambda$ от геометрических размеров жёстко закреплённой с обоих краёв оболочки $(C C)$ в случае невязкой $\left(v_{f}=0\right)$ и вязкой $\left(v_{f} \neq 0\right)$ жидкостей

ностью в задании параметра $s$, так и тем, что исследование [13] является аналитическим и профиль скорости жидкости по радиусу оболочки в нём не учитывается. При этом как в случае невязкой, так и вязкой жидкости увеличение длины и уменьшение толщины оболочки приводит к дестабилизации системы.

Далее рассматриваются системы горизонтально расположенных коаксиальных цилиндрических оболочек, состоящие из внутренней и внешней оболочек с радиусами $R^{(1)}$ и $R^{(2)}$ соответственно. Жидкость заполняет только кольцевой зазор между оболочками на высоту $H$. В данном исследовании влияние вязкости жидкости не учитывалось, поскольку роль уровня заполнения на скорости потери устойчивости не изучена даже в случае идеальной жидко- сти. Для представления результатов вводятся следующие безразмерные параметры уровня заполнения п и величины кольцевого зазора между оболочками $k$ :

$$
\eta=H /\left(2 R^{(2)}\right), \quad k=\left(R^{(2)}-R^{(1)}\right) / R^{(1)} .
$$

В случае частичного заполнения горизонтально ориентированных коаксиальных оболочек происходит расщепление собственных частот, вследствие чего двум формам, отвечающим одинаковым комбинациям волновых чисел, соответствуют разные частоты. Данный факт демонстрируется на рис. 7, где показаны зависимости мнимых (частота колебаний) и действительных (величина демпфирования системы) частей собственных значений $\lambda$ от безразмерного уровня заполнения $\eta$ кольцевого канала шириной $k=1 / 10$. Здесь внешняя оболочка является абсолютно жё-
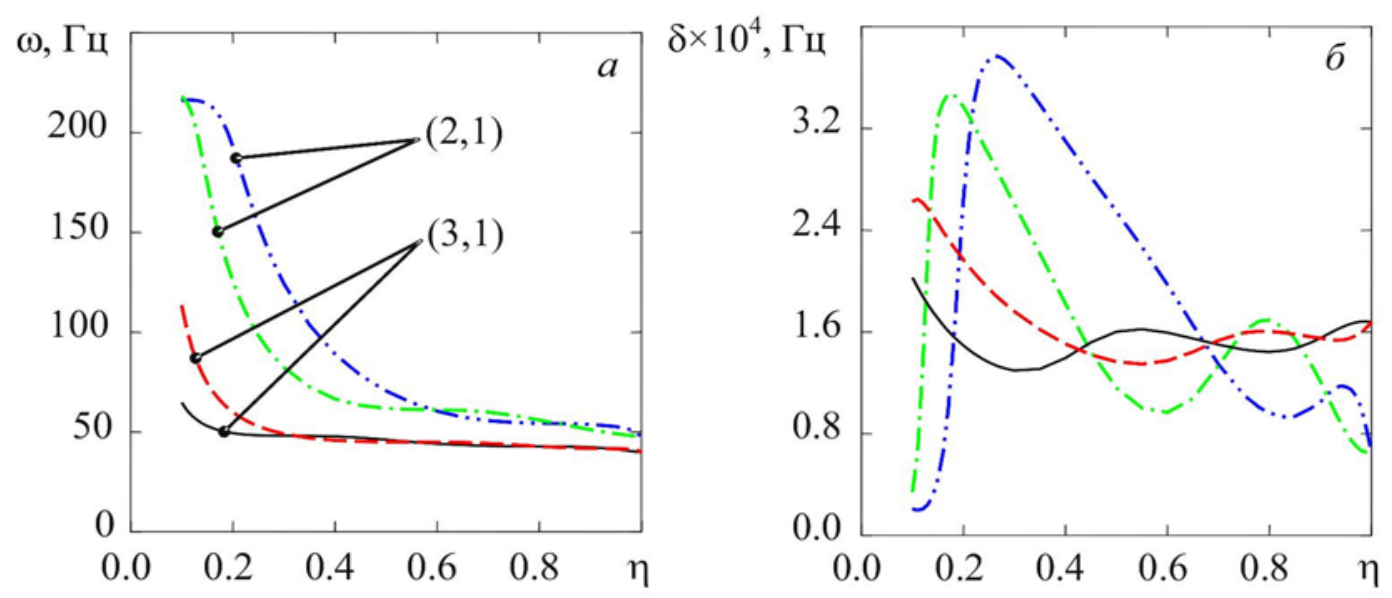

Рис. 7. Зависимости частоть (а) и величины демпфирования (б) от безразмерного уровня заполнения п для случая внешней жёсткой оболочки $(k=1 / 10, C С)$ 
сткой, а упругая внутренняя оболочка жёстко закреплена с обоих краёв. Симметричная и антисимметричная компоненты частот совпадают, когда канал между оболочками заполнен полностью $(\eta=1)$. При снижении уровня жидкости $(\eta<1)$ они начинают отличаться друг от друга, что характеризует расщепление частот на ветви. Другой предельный случай соответствует оболочкам с воздушной средой в зазоре $(\eta=0)$. В таких условиях частотный спектр будет наиболее близок к несвязанным колебаниям разных оболочек в вакууме, и различные ветви должны совпасть. На рис. 8 изображены зависимости низших частот $\omega(a)$ и безразмерных скоростей потери устойчивости $\Lambda(\sigma)$ от безразмерного уровня заполнения $\eta$, полученные при различных значениях кольцевого зазора $k$ между двумя упругими оболочками. На при- ведённых графиках показано, что понижение уровня жидкости приводит к росту обеих величин. Это обусловлено уменьшением площади смоченных поверхностей и снижением роли присоединённой массы жидкости. Резкое изменение низшей частоты $\omega$ критической скоростей потери устойчивости $\Lambda$ наблюдается при малом уровне заполнения $\eta$ для зазора $k=1 / 100$. Данное явление возникает только при узких зазорах. Оно обусловлено необходимостью существования смоченной поверхности внутренней оболочки, которая может отсутствовать при малом количестве жидкости в относительно широком кольцевом канале. Волнообразный характер кривых на рис. 8 связан с чередованием минимальных значений, отвечающих формам колебаний с одинаковой комбинацией волновых чисел.
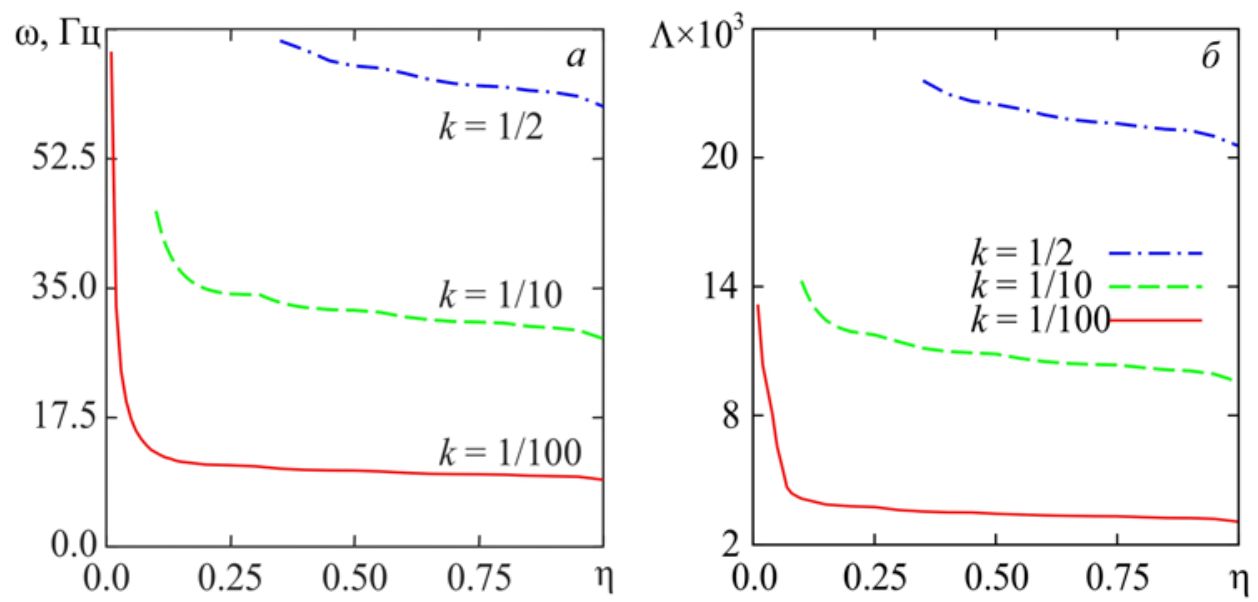

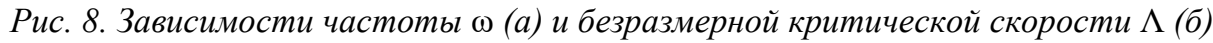
от безразмерного уровня заполнения п кольцевого канала при пустой внутренней оболочке для упругих оболочек, СС

\section{Библиографический список}

1. Бочкарёв С.А., Лекомиев С.В., Сенин А.Н. Анализ пространственных колебаний коаксиальных цилиндрических оболочек, частично заполненных жидкостью // Вычислительная механика сплошных сред. - 2018. - Т. 11. - № 4. - С. 448-462.

2. Вольмир А.С. Оболочки в потоке жидкости и газа. Задачи гидроупругости. - М.: Наука, 1979. - 320 с.

3. Гузь А.Н. Проблемы гидроупругости для сжимаемой вязкой жидкости // Прикладная механика. 1991. - Т. 27. - № 1. - С. 3-15.

4. Зенкевич О.С. Метод конечных элементов в технике. - М.: Мир, 1975. -544 с.

5. Ильгамов М.A. Колебания упругих оболочек, содержащих жидкость и газ. - М.: Наука, 1969. - 182 с.

6. Кондратов Д.В., Могилевич Л.И. Математическое моделирование процессов взаимодействия двух цилиндрических оболочек со слоем жидкости между ними при отсутствии торцевого истечения в условия вибрации // Вестник СГТУ. - 2007. - Т. 3. - № 2. - С. 15-23.

7. Шлихтинг Г. Теория пограничного слоя. - М.: Наука, 1974. - 712 с.

8. Amabili M., Garziera R. Vibrations of circular cylindrical shells with nonuniform constraints, elastic bed and added mass; Part II: Shells containing or immersed in axial flow // J. Fluids Struct. - 2002. - Vol. 16. P. 31-51. 
9. Amabili M., Garziera R. Vibrations of circular cylindrical shells with nonuniform constraints, elastic bed and added mass; Part III: steady viscous effects on shells conveying fluid // J. Fluids Struct. - 2002. Vol. 16. - P. 795-809.

10. Bochkarev S., Kamenskikh A., Lekomtsev S. Experimental and numerical investigation of eigenfrequencies of rectangular plates, interacting with a fluid // MATEC Web Conf. - 2018. - Vol. 148. - 07002.

11. Bochkarev S.A., Matveenko V.P. Stability analysis of loaded coaxial cylindrical shells with internal fluid flow // Mech. Sol. - 2010. - Vol. 45. - P. 789-802.

12. Bochkarev S.A., Matveenko V.P. The dynamic behaviour of elastic coaxial cylindrical shells conveying fluid // J. Appl. Math. Mech. - 2010. - Vol. 74. - P. 467-474.

13. El Chebair A., Misra A.K., Païdoussis M.P. Theoretical study of the effect of unsteady viscous forces on inner- and annular-flow-induced instabilities of cylindrical shells // J. Sound Vib. - 1990. - Vol. 138. - № 3. P. 457-478.

14. El Chebair A., Païdoussis M.P., Misra A.K. Experimental study of annular-flow-induced instabilities of cylindrical shells // J. Fluids Struct. - 1989. - Vol. 3. -P. 349-364.

15. Horáček J., Trnka J., Veselý J., Gorman D.G. Vibration analysis of cylindrical shells in contact with an annular fluid region // Eng. Struct. - 1995. - Vol. 17. - № 10. - P. 714-724.

16. Horáček J., Zolotarev I. Free vibration and stability of cylindrical shells in interaction with flowing fluid // In: Pellicano F., Mikhlin Y., Zolotarev I., NATO CLG Grant Report No. PST.CLF.977350. - Prague: F. Institute of Thermomechanics, 2002. - P. 45-82.

17. Joseph D.D. Viscous potential flow // J. Fluid Mech. - 2003. - Vol. 479. - P. 191-197.

18. Joseph D., Funada T., Wang J. Potential flows of viscous and viscoelastic fluids. - Cambridge: Cambridge University Press, 2008. - 516 p.

19. Lehoucq R.B., Sorensen D.C. Deflation techniques for an implicitly restarted Arnoldi iteration // SIAM J. Matrix Anal. Appl. - 1996. - Vol. 17. - № 4. - P. 789-821.

20. Mokeyev V.V. On a method for vibration analysis of viscous compressible fluid-structure systems // Int. J. Num. Meth. Eng. - 2004. - Vol. 59. - № 13. - P. 1703-1723.

21. Nguyen V.B., Païdoussis M.P., Misra A.K. A CFD-based model for the study of the stability of cantilevered coaxial cylindrical shells conveying viscous fluid // J. Sound Vib. - 1994. - Vol. 176. - P. 105-125.

22. Nguyen V.B., Païdoussis M.P., Misra A.K. An experimental study of the stability of cantilevered coaxial cylindrical shells conveying fluid // J. Fluids Struct. - 1993. - Vol. 7. - P. 913-930.

23. Ning W.B., Wang D.Z. Dynamic and stability response of a cylindrical shell subjected to viscous annular flow and thermal load // Int. J. Str. Stab. Dyn. - 2016. -Vol. 16. - 1550072.

24. Ning W.B., Wang D.Z., Zhang J.G. Dynamics and stability of a cylindrical shell subjected to annular flow including temperature effects // Arch. Appl. Mech. - 2016. - Vol. 86. - P. 643-656.

25. Ning W.-B., Xu Y., Liao Y.-H., Li Z.-R. Effects of geometric parameters on dynamic stability of the annular flow-shell system // Proceedings of the 3rd Annual International Conference on Mechanics and Mechanical Engineering (MME 2016). - Atlantis Press, 2017. - Vol. 150. - P. 344-350.

26. Païdoussis M.P. Fluid-Structure Interactions: Slender Structures and Axial Flow, Vol. 1, 2nd ed. - London: Elsevier Academic Press, 2014. - 888 p.

27. Païdoussis M.P. Fluid-Structure Interactions: Slender Structures and Axial Flow, Vol. 2, 2nd ed. - London: Elsevier Academic Press, 2016. - 942 p.

28. Païdoussis M.P., Misra A.K., Chan S.P. Dynamics and stability of coaxial cylindrical shells conveying viscous fluid // Appl. Mech. - 1985. - Vol. 52. - P. 389-396.

29. Païdoussis M.P., Misra A.K., Nguyen V.B. Internal- and annular-flow-induced instabilities of a clampedclamped or cantilevered cylindrical shell in a coaxial conduit: the effects of system parameters // J. Sound Vib. - 1992. - Vol. 159. - P. 193-205.

30. Païdoussis M.P., Nguyen V.B., Misra A.K. A theoretical study of the stability of cantilevered coaxial cylindrical shells conveying fluid // J. Fluids Struct. - 1991. - Vol. 5. - P. 127-164.

31. Reddy J.N. An introduction to nonlinear finite element analysis. 2nd Ed. - Oxford: Oxford University Press, 2015. $-687 \mathrm{p}$.

32. Yeh T.T. Chen S.S. Dynamics of a cylindrical shell system coupled by viscous fluid // J. Acoust. Soc. Am. 1977. - Vol. 62. - № 2. - P. 262-270.

33. Yeh T.T., Chen S.S. The effect of fluid viscosity on coupled tube/fluid vibrations // J. Sound Vib. - 1978. Vol. 59. - № 53. - P. 453-467. 


\title{
THEORETICAL AND EXPERIMENTAL RESEARCH OF THIN-WALLED STRUCTURES INTERACTING WITH VISCOUS FLUID
}

\author{
S.A. Bochkarev, A.O. Kamenskikh, S.V. Lekomtsev, A.N. Senin \\ Institute of Continuous Media Mechanics RAS
}

The results of studying thin-walled plates and cylindrical shells interacting with the stationary or flowing viscous compressible fluid are presented. The numerical solution of the problem has been carried out using the finite element method. The motion of liquid medium is described by the system of linearized Navier-Stokes equations, the solution of which is sought for in the form of the acoustic approximation in terms of velocity perturbation potential. The relations obtained for liquid and the corresponding boundary conditions are transformed by the Bubnov-Galerkin method. The behavior of a thin-walled structure is described in the framework of the classical theory of thin plates. The mathematical formulation of the dynamic problem of an elastic body is based on the variational principle of virtual displacements. Stability estimation is based on the calculation and analysis of complex eigenvalues of a coupled system of equations. The influence of fluid viscosity and other parameters on the natural vibration frequencies and critical velocities responsible for the loss of structural stability has been analyzed. The natural frequencies and the corresponding decrements of harmonic vibrations of rectangular plates located in the air and on the free liquid surface have been studied with the help of developed experimental setup. It has been found out that the damping coefficient corresponding to one vibration mode (bending or torsional) grows with an increase in the number of nodal lines. It has been demonstrated that this relation can be violated during the interaction of the plates with the liquid.

Keywords: viscous potential fluid, thin-walled structures, stability, natural vibrations, decrement of vibrations, FEM.

\section{Сведения об авторах}

Бочкарев Сергей Аркадьевич, кандидат физико-математических наук, старший научный сотрудник, Институт механики сплошных сред УрО РАН - филиал Пермского федерального исследовательского центра УрО РАН (ИМСС УрО РАН), 614013, г. Пермь, ул. Академика Королёва, e-mail: bochkarev@icmm.ru

Каменских Александр Олегович, инженер, ИМСС УрО РАН, 614013, г. Пермь, ул. Академика Королёва, 1; e-mail: sanyakamen96@mail.ru

Лекомиев Сергей Владимирович, кандидат физико-математических наук, старший научный сотрудник, ИМСС УрО РАН, 614013, г. Пермь, ул. Академика Королёва, 1; e-mail: lekomtsev@icmm.ru

Сенин Александр Николаевич, младший научный сотрудник, ИМСС УрО РАН, 614013, г. Пермь, ул. Академика Королёва, 1; e-mail: senin.a@icmm.ru 